\title{
Mathematics
}

Citation: Pathan MA, Kumar H. On A Logarithmic Mittag-Leffler Function, its Properties and Applications. Rev. Acad. Colomb. Cienc. Ex. Fis. Nat. 45(176):901-915, julio-septiembre de 2021. doi: https://doi.org/10.18257/ raccefyn. 1325

Editor: Jorge Cossio

*Corresponding autor:

M. A. Pathan; mapathan@gmail.com

Received: October 19, 2020

Accepted: May 20, 2021

Published: September 17, 2021

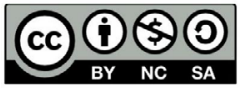

Este artículo está bajo una licencia de Creative Commons ReconocimientoNoComercial-Compartir Igual 4.0 Internacional
Original article

\section{On A Logarithmic Mittag-Leffler Function, its Properties and Applications}

\author{
Sobre una función logarítmica de Mittag-Leffler, sus \\ propiedades y aplicaciones
}

\author{
M. A. Pathan ${ }^{1, *}$, Hemant Kumar ${ }^{2}$ \\ ${ }^{1}$ Centre for Mathematical and Statistical Sciences, Peechi Campus, Peechi-680653, Kerala, India \\ ${ }^{2}$ Department of Mathematics, D. A-V. Postgraduate College Kanpur-208001, Uttar Pradesh, India
}

\begin{abstract}
In this paper, we introduce a logarithmic Mittag-Leffler function and discuss some of its properties. The application of these properties become helpful in extension of Pochhammer's type contour integral representations and Rodrigues formulae of some known hypergeometric functions. On application point of view, some relations are discussed which are useful in interpreting the phenomenon of spread of infectious diseases in terms of Lauricella's multiple hypergeometric functions.
\end{abstract}

2010Mathematics Subject Classification: 33E12, 33A17, 34A08.

Keywords: Logarithmic Mittag-Leffler function, complex order derivative, extended Pochhammer's type integrals, Rodrigues formulae, hypergeometric functions, spread of infectious diseases.

\section{Resumen}

En este artículo, presentamos una función logarítmica de Mittag-Leffler y discutir algunas de sus propiedades. La aplicación de estas propiedades se vuelven útiles en la extensión de la integral de contorno de tipo de Pochhammer representaciones y fórmulas de Rodrigues de algunos conocidos hipergeométricos funciones. Desde el punto de vista de la aplicación, se discuten algunas relaciones que son útiles para interpretar el fenómeno de la propagación de infecciones enfermedades en términos de las múltiples funciones hipergeométricas de Lauricella.

Palabras clave: Función logarítmica de Mittag-Leffler, orden complejo derivada, integrales de tipo de Pochhammer extendidas, fórmulas de Rodrigues, funciones hipergeométricas, propagación de enfermedades infecciosas.

\section{Introduction}

The study of classical Mittag-Leffler functions ((Gorenflo, Loutchko, \& Luchko, 2002), (Gorenflo, Luchko, \& Mainardi, 1999), (Mittag-Leffler, 1903), (Wiman, 1905)) has tremendous applications in solving many of the scientific problems involving fractional derivatives and integrals (for example (Mathai \& Haubold, 2008), (Oldham \& Spanier, 2006) and (Pathan \& Kumar, 2019)). In this presentation, we consider a transformation formula involving the logarithm of Mittag- Leffler function in the form: 


$$
\begin{gathered}
\zeta_{m}^{(\alpha)}(z)=A^{(\alpha)} \log _{e} E_{\frac{1}{m}}\left(z^{\frac{1}{m}}\right)=A^{(\alpha)} \log E_{\frac{1}{m}}\left(z^{\frac{1}{m}}\right), z \in \mathbb{C}, A^{(\alpha)}=\frac{1}{\log _{e} E_{\alpha}}, \alpha \in \mathbb{R}, \alpha>0, \\
\forall m \in \mathbb{N}^{*}, \mathbb{N}^{*}=\{2,3,4, \ldots, L\}, L<\infty ; E_{\alpha}(z)=\sum_{k=0}^{\infty} \frac{z^{k}}{\Gamma(\alpha k+1)}, E_{\alpha} \equiv E_{\alpha}(1) .
\end{gathered}
$$

By simple logarithmic rulings, the function (1.1) is written in the form

$$
\zeta_{m}^{(\alpha)}(z)=\log _{E_{\alpha}} E_{\frac{1}{m}}\left(z^{\frac{1}{m}}\right), \text { or } \zeta_{m}^{(\alpha)}(z)=\log _{e}\left\{E_{\frac{1}{m}}\left(z^{\frac{1}{m}}\right)\right\}^{\frac{1}{\log _{e} E_{\alpha}}},
$$

where all conditions given in (1.1) are followed.

Further, we find the inequality of $E_{\alpha}$ greater than as

$$
\begin{array}{r}
E_{\alpha}=\sum_{k=0}^{\infty} \frac{1}{\Gamma(\alpha k+1)}=1+\frac{1}{\Gamma(\alpha+1)}+\frac{1}{\Gamma(2 \alpha+1)}+\frac{1}{\Gamma(3 \alpha+1)}+\frac{1}{\Gamma(4 \alpha+1)}+\ldots \\
\Rightarrow E_{\alpha}>\frac{1+\Gamma(\alpha+1)}{\Gamma(\alpha+1)}
\end{array}
$$

Again, we know that $\Gamma(3 \alpha+1) \sim\left(\frac{3 \alpha}{e}\right)^{3 \alpha} \sqrt{2 \pi(3 \alpha)}$ and $\{\Gamma(2 \alpha+1)\}^{2} \sim\left(\frac{2 \alpha}{e}\right)^{4 \alpha}(\pi(4 \alpha))$. Also we have, $\frac{\Gamma(3 \alpha+1)}{\{\Gamma(2 \alpha+1)\}^{2}}=\frac{\left(\frac{3 \alpha}{e}\right)^{3 \alpha} \sqrt{2 \pi(3 \alpha)}}{\left(\frac{2 \alpha}{e}\right)^{4 \alpha}(\pi(4 \alpha))}>1, \forall \alpha \in \mathbb{R}, \alpha>0$, so that $\Gamma(3 \alpha+1)>(\Gamma(2 \alpha+$ 1) $)^{2}$.

Similarly, we find that

$$
\Gamma(4 \alpha+1)>(\Gamma(2 \alpha+1))^{3}, \Gamma(5 \alpha+1)>(\Gamma(2 \alpha+1))^{4}, \ldots,
$$

(these results are verified by MATLAB for $\forall \alpha \in \mathbb{R}, \alpha>0$ ).

Thus, we obtain the inequality of $E_{\alpha}$ less than by the series as

$$
\begin{gathered}
E_{\alpha}<1+\frac{1}{\Gamma(\alpha+1)}+\frac{1}{\Gamma(2 \alpha+1)}+\left\{\frac{1}{\Gamma(2 \alpha+1)}\right\}^{2}+\left\{\frac{1}{\Gamma(2 \alpha+1)}\right\}^{3}+\left\{\frac{1}{\Gamma(2 \alpha+1)}\right\}^{4}+\ldots \\
=\frac{\{\Gamma(2 \alpha+1)-1\}+\{\Gamma(2 \alpha+1) \Gamma(\alpha+1)\}}{\{\Gamma(\alpha+1)\}\{\Gamma(2 \alpha+1)-1\}} .
\end{gathered}
$$

Therefore, the value of $E_{\alpha}$ bounds between the numbers

$$
\frac{\Gamma(\alpha+1)+1}{\Gamma(\alpha+1)}<E_{\alpha}<\frac{\{\Gamma(2 \alpha+1)-1\}+\{\Gamma(2 \alpha+1) \Gamma(\alpha+1)\}}{\{\Gamma(\alpha+1)\}\{\Gamma(2 \alpha+1)-1\}}, \forall \alpha \in \mathbb{R}, \alpha>0 .
$$

Particularly, when $\alpha=1$, the inequality (1.3) becomes well known inequality $2<e<3$.

Some other special cases of Mittag - Leffler function $E_{\alpha}(z)$ (see (Gorenflo et al., 2002), (Gorenflo et al., 1999) and (Peng \& Li, 2010) and the earlier papers cited therein ) are

$$
E_{1}(z)=e^{z}, E_{1}(1)=E_{1}=e, E_{\frac{1}{2}}\left(z^{\frac{1}{2}}\right)=e^{z} \operatorname{erfc}(-\sqrt{z}),
$$

where,

$$
\operatorname{erfc}(z)=\frac{2}{\sqrt{\pi}} \int_{z}^{\infty} e^{-u^{2}} d u
$$

and $\lim _{\alpha \rightarrow 0} E_{\alpha}(z)=\frac{1}{1-z}$, so that

$$
\lim _{\substack{\alpha \rightarrow 0 \\ z \rightarrow 1}} E_{\alpha}(z) \rightarrow \infty
$$


Therefore by the relations of Mittag - Leffler functions given in (1.3), the function $\zeta_{m}^{(\alpha)}(z)$ defined in (1.1) and (1.2), gives us the values

$$
\zeta_{1}^{(\alpha)}(z)=\frac{z}{\log _{e} E_{\alpha}} \text { and } \lim _{m \rightarrow \infty} \zeta_{m}^{(\alpha)}(z) \rightarrow \infty, \quad \alpha>0 .
$$

\section{I) To prove that}

$$
\zeta_{1}^{(\alpha)}=\frac{z}{\log _{e} E_{\alpha}}, \alpha>0
$$

Proof. For $\alpha>0$, consider the formula $\zeta_{m}^{(\alpha)}=\log _{E_{\alpha}} E_{\frac{1}{m}}\left(z^{\frac{1}{m}}\right)$ given in (1.2), and use the logarithmic formula $\log _{a} b=\frac{\log _{e} b}{\log _{e} a}$, we write

$$
\zeta_{1}^{(\alpha)}=\log _{E_{\alpha}} E_{1}(z)=\frac{\log _{e} E_{1}(z)}{\log _{e} E_{\alpha}},
$$

finally, on using the relations $E_{1}(z)=e^{z}, \log _{e} e=1$, we find

$$
\zeta_{1}^{(\alpha)}=\frac{\log _{e} E_{1}(z)}{\log _{e} E_{\alpha}}=\frac{z \log _{e} e}{\log _{e} E_{\alpha}}=\frac{z}{\log _{e} E_{\alpha}} .
$$

Therefore, we have

$$
\zeta_{1}^{(\alpha)}=\frac{z}{\log _{e} E_{\alpha}}, \alpha>0
$$

\section{II) Again, to prove that}

$$
\lim _{m \rightarrow \infty} \zeta_{m}^{(\alpha)} \rightarrow \infty
$$

Proof. Use the relations

$\lim _{m \rightarrow \infty} \log _{e} E_{\frac{1}{m}}\left(z^{\frac{1}{m}}\right)=\lim _{z \rightarrow 1} \log _{e} E_{0}(z), E_{0}(z)=\frac{1}{1-z}$ in the Eqn. (1.2) and by above logarithmic formula given in I), we find that

$$
\lim _{m \rightarrow \infty} \zeta_{m}^{(\alpha)}=\frac{1}{\log _{e} E_{\alpha}} \lim _{m \rightarrow \infty} \log _{e} E_{\frac{1}{m}}\left(z^{\frac{1}{m}}\right)=\frac{1}{\log _{e} E_{\alpha}} \lim _{z \rightarrow 1} \log _{e}\left(\frac{1}{1-z}\right) .
$$

Thus there exists following relations

$$
\lim _{m \rightarrow \infty} \zeta_{m}^{(\alpha)}=\frac{1}{\log _{e} E_{\alpha}} \lim _{z \rightarrow 1}\left[z+\frac{z^{2}}{2}+\frac{z^{3}}{3}+\ldots+\frac{z^{n}}{n}+\ldots\right]=\frac{1}{\log _{e} E_{\alpha}} \lim _{n \rightarrow \infty}\left[1+\frac{1}{2}+\frac{1}{3}+\ldots+\frac{1}{n}\right] .
$$

Therefore, we may write

$$
\lim _{m \rightarrow \infty} \zeta_{m}^{(\alpha)}=\frac{1}{\log _{e} E_{\alpha}} \lim _{n \rightarrow \infty}\left[\left\{1+\frac{1}{2}+\frac{1}{3}+\ldots+\frac{1}{n}-\log n\right\}+\log n\right] .
$$

Now, here use the relation of Euler - Mascheroni constant $\gamma$, (H. M. Srivastava \& Manocha, 1984, p. 20) given by

$\gamma=\lim _{n \rightarrow \infty}\left\{1+\frac{1}{2}+\frac{1}{3}+\ldots+\frac{1}{n}-\log n\right\} \cong 0.5772156649 \ldots$, we write

$$
\lim _{m \rightarrow \infty} \zeta_{m}^{(\alpha)}=\frac{1}{\log _{e} E_{\alpha}} \lim _{n \rightarrow \infty}[\gamma+\log n] \rightarrow \infty .
$$


In order to discuss some more properties of Mittag - Leffler function $E_{\alpha}$, we prefer to use the Mellin-Barnes integral formula given by (Mathai \& Haubold, 2008, p. 88)

$$
E_{\alpha}(z)=\frac{1}{2 \pi i} \int_{c-i \infty}^{c+i \infty} \frac{\Gamma(s) \Gamma(1-s)}{\Gamma(1-\alpha s)}(-z)^{-s} d s, 0<c<1, s \in \mathbb{C}
$$

and

$$
\alpha \in \mathbb{R}, \alpha>0,|\arg (z)|<\pi, i=\sqrt{(-1)}, \text { in throughout this paper. }
$$

Also, we determine the analyticity of logarithmic Mittag -Leffler function $\zeta_{m}^{(\alpha)}(z)$ and introduce the theory and concept of derivative of complex order defined by Campos (Campos, 1984 ) as the derivative of complex order $v$ of complex function $f(z)$ with respect to the complex variable $z$. Next, we apply fractional calculus theory and obtain extended Pochhammer's type integral representations and Rodrigues formulae (Oldham \& Spanier, 2006, pp. 186 -192) of some known special functions through their complex order derivatives. In a compartment method, to obtain the area of spread of infectious disease, it is supposed that the area spread depends on various parameters with weight functions. A quite different approach using a property of the logarithmic Mittag - Leffler function, we obtain the spread area of infectious diseases for different weight functions and parameters in terms of Lauricella's multiple hypergeometric functions (H. M. Srivastava \& Manocha, 1984, p. 60). It is noted that various distinguished roles of the Mittag-Leffler function and its properties are described in the researches ((Kilbas, Srivastava, \& Trujillo, 2006) - (Kiryakova \& Luchko, 2010), (Rogosin, 2015) - (B. L. Srivastava, Pathan, \& Kumar, 2020)) and its generalizations in fractional analysis and fractional modeling are analysed in the work of researchers ((Caponetto, Dongola, \& L. Fortuna, 2010), (Diethelm, 2010), (Dzherbashian, 1966), (Gorenflo, Kilbas, Mainardi, \& Rogosin, 2014), (Gorenflo, Mainardi, \& Srivastava, 1998), (Hilfer, 2000), (Osler, 1972) and (Podlubny, 1999)).

\section{Analyticity of logarithmic Mittag -Leffler function $\zeta_{m}^{(\alpha)}(z)$}

To determine the analyticity of logarithmic Mittag -Leffler function $\zeta_{m}^{(\alpha)}(z)$, we present following lemmas and theorems:

Lemma 2.1. If $s \in \mathbb{C}$ and $\alpha \in \mathbb{R}, \alpha>0$, then for $0<c<1$, the Mellin-Barnes type integral of $E_{\alpha}$ is given by

$$
E_{\alpha}=\frac{1}{2 \pi i} \int_{c-i \infty}^{c+i \infty} \frac{\Gamma(s) \Gamma(1-s)}{\Gamma(1-\alpha s)}\left(e^{\pi i}\right)^{-s} d s
$$

Proof. The contour integral in (2.1) separates all poles $s=-k, k=0,1,2, \ldots$ to the left and all poles $s=n+1, n=0,1,2, \ldots$ to the right. Hence, by calculus of residue $\left(\lim _{s \rightarrow-k}(s+\right.$ $k) \Gamma(s)=\frac{(-1)^{k}}{k !}$ and the sum of the residue, at the points $s=0,-1,-2, \ldots$ of the contour integral in (2.1), we write

$$
\begin{array}{r}
\frac{1}{2 \pi i} \int_{c-i \infty}^{c+i \infty} \frac{\Gamma(s) \Gamma(1-s)}{\Gamma(1-\alpha s)}(-1)^{-s} d s=\sum_{k=0}^{\infty} \lim _{s \rightarrow-k} \frac{(s+k) \Gamma(s) \Gamma(1-s)}{\Gamma(1-\alpha s)} \\
=\sum_{k=0}^{\infty} \frac{(-1)^{k}}{k !} \frac{\Gamma(1+k)}{\Gamma(1+\alpha k)}(-1)^{k}
\end{array}
$$

Now, using the definition of $E_{\alpha}(z)$ given in (1.1) with $z=1$, (2.2) becomes

$$
\frac{1}{2 \pi i} \int_{c-i \infty}^{c+i \infty} \frac{\Gamma(s) \Gamma(1-s)}{\Gamma(1-\alpha s)}\left(e^{\pi i}\right)^{-s} d s=E_{\alpha} .
$$

Hence, the result (2.1) is found. This result is obviously identical to the formula (1.5) (when, $z=1$ ). 
Set $\alpha=1$ in the formula (2.1) to get the Mellin - Barnes type integral for $E_{1}=e$ as

$$
E_{1}=e=\frac{1}{2 \pi i} \int_{c-i \infty}^{c+i \infty} \Gamma(s)\left(e^{\pi i}\right)^{-s} d s, \text { when } 0<c<1 .
$$

Lemma 2.2. If $f(z)$ is holomorphic on a simply connected domain $\mathbb{D} \subset \mathbb{C}$ and $0 \notin f(\mathbb{D})$ then there exists another function $g(z)=\log f(z)$, holomorphic on $\mathbb{D}$ and $f(\mathbb{D})=\mathbb{C} \backslash\{0\}$ is not simply connected.

Proof. By the relation $g(z)=\log f(z)$, we may write $f(z)=\exp [g(z)]=\exp [g(z)+2 n \pi i] \forall n=$ $0,1,2, \ldots$ Since there is no complex number $[g(z)+2 n \pi i] \in \mathbb{C} \forall n=0,1,2, \ldots$ so that $f(z)$ becomes zero, hence $0 \notin f(\mathbb{C}) \forall z \in \mathbb{D}=\mathbb{C}$. Again, $\mathbb{D}$ is simply connected, then, there exists $\frac{d}{d z} g(z)=\frac{\frac{d}{d z} f(z)}{f(z)}:=g^{\prime}(z)=\frac{f^{\prime}(z)}{f(z)}$. Thus we have

$$
g(z)=\int_{z_{0}}^{z} \frac{f^{\prime}(\xi)}{f(\xi)} d \xi
$$

Now in (2.5), for example set $f(z)=e^{z}$, then by the relation $g(z)=\log f(z)$, the left hand side of (2.5) becomes $z$, and that of right hand side is $z-z_{0}$ and thus ultimately, the result is contradiction. Hence $g(z)$ could not be defined for a continuous single-valued branch of $\log f(z)$. Therefore in this case $f(\mathbb{D})=\mathbb{C} \backslash\{0\}$ is not simply connected.

Lemma 2.3. If $f(z)$ is an entire function $\forall z \in \mathbb{C}$, in the complex plane, then there exists another function $G(z)=e^{-z} \log f(z)$ analytic $\forall z \in \mathbb{C}$ in that complex plane.

Proof. By the relation $G(z)=e^{-z} \log f(z)$, we have $\log f(z)=e^{z} G(z)$ so that by Lemma 2.2, we get

$$
\int_{z_{0}}^{z} \frac{f^{\prime}(\xi)}{f(\xi)} d \xi=\left.\left(e^{\xi} G(\xi)\right)\right|_{\xi=z_{0}} ^{\xi=z}=\log f(z)-\log f\left(z_{0}\right) .
$$

Now for the complex analysis involved, we refer Conway (Conway, 1973). It follows immediately that the proof of the Lemma 2.3 is trivial. To verify the equality, we set $f(z)=$ $e^{z}$ in both sides of this result and the Lemma is followed.

For another example, set $f(z)=E_{\frac{1}{m}}\left(z^{\frac{1}{m}}\right), \forall m \in \mathbb{N}^{*}, \mathbb{N}^{*}=\{2,3,4, \ldots, L\}, L<\infty$, in Lemma 2.3 , then we have an analytic function

$$
G(z)=e^{-z} \log E_{\frac{1}{m}}\left(z^{\frac{1}{m}}\right) .
$$

Now on differentiating both the sides of (2.7), we have

$$
e^{-z} \frac{d}{d z} E_{\frac{1}{m}}\left(z^{\frac{1}{m}}\right)=e^{-z} E_{\frac{1}{m}}\left(z^{\frac{1}{m}}\right)+\frac{d}{d z}\left(e^{-z} E_{\frac{1}{m}}\left(z^{\frac{1}{m}}\right)\right)
$$

which is a linear differential equation

$$
\frac{d}{d z} G(z)+G(z)=\frac{\frac{d}{d z}\left(e^{-z} E_{\frac{1}{m}}\left(z^{\frac{1}{m}}\right)\right)}{E_{\frac{1}{m}}\left(z^{\frac{1}{m}}\right)}+e^{-z}
$$

whence, upon integration, we obtain an integral function

$$
G(z)=e^{-z} \int\left\{\frac{d}{d z} \log \left(e^{-z} E_{\frac{1}{m}}\left(z^{\frac{1}{m}}\right)\right)\right\} d z+z e^{-z}+C,
$$


$C$ is arbitrary constant, or its equivalent form

$$
G(z)=e^{-z} \log \left(e^{-z} E_{\frac{1}{m}}\left(z^{\frac{1}{m}}\right)\right)+z e^{-z}+C,
$$

$C$ is arbitrary constant.

But, by (2.7) $G(0)=0$ and $\lim _{z \rightarrow 0} \log \left(e^{-z} E_{\frac{1}{m}}\left(z^{\frac{1}{m}}\right)\right)=0$. Thus $C=0$ and we find that

$$
G(z)=e^{-z} \log \left(e^{-z} E_{\frac{1}{m}}\left(z^{\frac{1}{m}}\right)\right)+z e^{-z} .
$$

Now multiply both sides of (2.8) by $A^{(\alpha)}$ and then use (1.1) and (1.2) to get a valuable relation

$$
\zeta_{m}^{(\alpha)}(z)=\chi_{m}^{(\alpha)}(z)+A^{(\alpha)} z, \forall z \in \mathbb{C}, \alpha \in \mathbb{R}, \alpha>0, m \in \mathbb{N}^{*}, \mathbb{N}^{*}=\{2,3,4, \ldots, L\}, L<\infty
$$

where

$$
\chi_{m}^{(\alpha)}(z)=A^{(\alpha)} \log \left\{e^{-z} E_{\frac{1}{m}}\left(z^{\frac{1}{m}}\right)\right\}
$$

Again then, letting $\Omega_{m}^{(\alpha)}(z)=\zeta_{m}^{(\alpha)}(z)-\chi_{m}^{(\alpha)}(z)$ in (2.9),we have

$$
\frac{d}{d z} \Omega_{m}^{(\alpha)}(z)=A^{(\alpha)} \Rightarrow\left\{\Omega_{m}^{(\alpha)}(z)\right\}^{\prime} d z=A^{(\alpha)} d z
$$

and

$$
G(\xi, z)=\lim _{\xi \rightarrow z}\left\{\frac{\Omega_{m}^{(\alpha)}(\xi)-\Omega_{m}^{(\alpha)}(z)}{\xi-z}\right\}=A^{(\alpha)},
$$

where the equation $\Omega_{m}^{(\alpha)}(\xi)-\Omega_{m}^{(\alpha)}(z)=0$ has a single root $\xi=z$ in a complex $\xi-$ plane in a region $\mathbb{D}$.

Theorem 2.1. If $\Omega_{m}^{(\alpha)}(z)=\zeta_{m}^{(\alpha)}(z)-\chi_{m}^{(\alpha)}(z), \zeta_{m}^{(\alpha)}(z)$, and $\chi_{m}^{(\alpha)}(z)$ are given in (1.1) and (2.9), respectively, $\forall z \in \mathbb{D}-\partial \mathbb{D} \subset \mathbb{C}, \alpha \in \mathbb{R}, \alpha>0, m \in \mathbb{N}^{*}, \mathbb{N}^{*}=\{2,3,4, \ldots, L\}, L<\infty$, and there is a continuous function $G(\xi, z)=\lim _{\xi \rightarrow z}\left\{\frac{\Omega_{m}^{(\alpha)}(\xi)-\Omega_{m}^{(\alpha)}(z)}{\xi-z}\right\}=A^{(\alpha)}$, the equation $\Omega_{m}^{(\alpha)}(\xi)-\Omega_{m}^{(\alpha)}(z)=0$, in a region $\mathbb{D}$ of a complex $\xi$ - plane, has single root $\xi=z$, and on a regular arc $\partial \mathbb{D}$ of length $l$, where $\vartheta_{k}$ is a point on the regular arc $\partial \mathbb{D}$ lying between the points $\xi_{k-1}$ and $\xi_{k}$ and $\vartheta_{k}=\xi\left(\tau_{k}\right)=x\left(\tau_{k}\right)+i y\left(\tau_{k}\right) \forall t_{k-1} \leq \tau_{k} \leq t_{k} ; k=1,2, \ldots$, also $\lim _{n \rightarrow \infty}\left|\sum_{k=1}^{n}\left(\xi_{k}-\xi_{k-1}\right)\right|=\int_{\partial \mathbb{D}}|d \xi|=l$, then there exists an absolute value

$$
\left|\lim _{\xi \rightarrow z} \int_{\partial \mathbb{D}} G(\xi, z) d \xi\right| \leq\left|A^{(\alpha)}\right| l \text {. }
$$

Proof. Since in the Theorem 2.1, $\forall z \in \mathbb{D}-\partial \mathbb{D} \subset \mathbb{C}, \alpha \in \mathbb{R}, \alpha>0, m \in \mathbb{N}^{*}, \mathbb{N}^{*}=\{2,3,4, \ldots, L\}$, $L<\infty$, the equation $\Omega_{m}^{(\alpha)}(\xi)-\Omega_{m}^{(\alpha)}(z)=0$, in a region $\mathbb{D}$ of a complex $\xi$ - plane, has single root $\xi=z$, and on a regular arc $\partial \mathbb{D}$ of length $l$, where $\vartheta_{k}$ is a point on the regular arc $\partial \mathbb{D}$ lying between the points $\xi_{k-1}$ and $\xi_{k}$ and $\vartheta_{k}=\xi\left(\tau_{k}\right)=x\left(\tau_{k}\right)+i y\left(\tau_{k}\right) \forall t_{k-1} \leq \tau_{k} \leq t_{k} ; k=$ $1,2, \ldots$, so let the points $a=\xi_{0}<\xi_{1}<\ldots<\xi_{n}=b$ lie on the arc of length $l$ in the interval $(a, b) \subset(-\infty, \infty)$. Then by (2.10) and by Campos theory for complex derivatives (Campos, 1984), the left hand side of the integral of the equation (2.11) is written as

$$
\begin{gathered}
\left|\lim _{\xi \rightarrow z} \int_{\partial \mathbb{D}} G(\xi, z) d \xi\right|=\lim _{n \rightarrow \infty}\left|\sum_{k=1}^{n}\left[\lim _{\xi \rightarrow z}\left\{\frac{\Omega_{m}^{(\alpha)}(\xi)-\Omega_{m}^{(\alpha)}(z)}{\xi-z}\right\}\right]\left(\xi_{k}-\xi_{k-1}\right)\right| \\
\leq \lim _{n \rightarrow \infty} \sum_{k=1}^{n}\left|A^{(\alpha)}\right|\left|\xi_{k}-\xi_{k-1}\right| \leq\left|A^{(\alpha)}\right| \lim _{n \rightarrow \infty} \sum_{k=1}^{n}\left|\xi_{k}-\xi_{k-1}\right| \leq A^{(\alpha)} l .
\end{gathered}
$$

Hence, the theorem is followed. 


\section{Full proof of the inequality}

$$
\left|\lim _{\xi \rightarrow z} \int_{\partial D} G(\xi, z) d \xi\right| \leq\left|A^{(\alpha)}\right||l|
$$

Consider

$$
\left|\lim _{\xi \rightarrow z} \int_{\partial D} G(\xi, z) d \xi\right|=\lim _{n \rightarrow \infty}\left|\sum_{k=1}^{n}\left[\lim _{\xi \rightarrow z}\left\{\frac{\Omega_{m}^{(\alpha)}(\xi)-\Omega_{m}^{(\alpha)}(z)}{\xi-z}\right\}\right]\left(\xi_{k}-\xi_{k-1}\right)\right|
$$

By Eqns. (2.9) and (2.10), such that $\lim _{\xi \rightarrow z}\left\{\frac{\Omega_{m}^{(\alpha)}(\xi)-\Omega_{m}^{(\alpha)}(z)}{\xi-z}\right\}=\frac{d}{d z} \Omega_{m}^{(\alpha)}(z)=A^{(\alpha)}$, then we find

$$
\left|\lim _{\xi \rightarrow z} \int_{\partial D} G(\xi, z) d \xi\right| \leq\left|A^{(\alpha)}\right| \lim _{n \rightarrow \infty} \sum_{k=1}^{n}\left[\left|\xi_{k}-\xi_{k-1}\right|\right] \leq\left|A^{(\alpha)}\right|\left|\xi_{n}-\xi_{0}\right| \leq\left|A^{(\alpha)}\right||b-a|,
$$

and then, we get

$$
\left|\lim _{\xi \rightarrow z} \int_{\partial D} G(\xi, z) d \xi\right| \leq\left|A^{(\alpha)}\right||l|
$$

Theorem 2.2. If $f: \mathbb{R} \rightarrow \mathbb{R}$, defined by the formula $T(f)=\lim _{\xi \rightarrow x} \int_{-\infty}^{+\infty} f(\xi)\left\{\frac{\Omega_{m}^{(\alpha)}(\xi)-\Omega_{m}^{(\alpha)}(x)}{\xi-x}\right\}$ $d \xi$, then, for $\int_{-\infty}^{\infty}|f(\xi)| d \xi \leq M<\infty, \alpha \in \mathbb{R}, \alpha>0, \forall \xi, x \in(-\infty, \infty)$, there exists an estimate

$$
|T(f)| \leq\left\{l M\left|A^{(\alpha)}\right|\right\}^{\frac{1}{2}} .
$$

Proof. Starting with the inequality given in (1.1) and the inequality of the integral (2.11), and using the techniques for Cauchy Schwarz inequality in (Steele, 2004, p.108), the left hand side of (2.12) is written as

$$
\begin{aligned}
\left|\lim _{\xi \rightarrow x} \int_{-\infty}^{+\infty} f(\xi)\left\{\frac{\Omega_{m}^{(\alpha)}(\xi)-\Omega_{m}^{(\alpha)}(x)}{\xi-x}\right\} d \xi\right|^{2} & \\
& \leq\left|\int_{-\infty}^{+\infty}\left\{\left|(f(\xi))^{\frac{1}{2}}\right|\right\}^{2} d \xi \lim _{\xi \rightarrow x} \int_{-\infty}^{\infty}\left\{\left|\left(\left\{\frac{\Omega_{m}^{(\alpha)}(\xi)-\Omega_{m}^{(\alpha)}(x)}{\xi-x}\right\}\right)^{\frac{1}{2}}\right|\right\}^{2} d \xi\right|
\end{aligned}
$$

On applying the Theorem 2.1, we have

$$
\begin{aligned}
\left|\lim _{\xi \rightarrow x} \int_{-\infty}^{+\infty} f(\xi)\left\{\frac{\Omega_{m}^{(\alpha)}(\xi)-\Omega_{m}^{(\alpha)}(x)}{\xi-x}\right\} d \xi\right|^{2} \\
\quad \leq \int_{-\infty}^{\infty}|f(\xi)| d \xi \lim _{\xi \rightarrow x} \int_{-\infty}^{\infty}\left|\left\{\frac{\Omega_{m}^{(\alpha)}(\xi)-\Omega_{m}^{(\alpha)}(x)}{\xi-x}\right\}\right| d \xi \leq l M\left|A^{(\alpha)}\right| .
\end{aligned}
$$

In this way, we obtain the estimate

$$
\left|\lim _{\xi \rightarrow x} \int_{-\infty}^{+\infty} f(\xi)\left\{\frac{\Omega_{m}^{(\alpha)}(\xi)-\Omega_{m}^{(\alpha)}(x)}{\xi-x}\right\} d \xi\right| \leq\left\{l M\left|A^{(\alpha)}\right|\right\}^{\frac{1}{2}} .
$$

Hence, inequality of the Theorem 2.2 is established. 


\section{Campos theory and extended derivative of complex function $f(z)$ with respect to a complex function $\Omega_{m}^{(\alpha)}(z)$}

In this section, we introduce the theory and concept of derivative of complex order defined by Campos (Campos, 1984, Eqn. (1), p. 113) as the derivative of complex order $v$ of complex function $f(z)$ with respect to $z$, the complex variable, in the form

$$
\begin{aligned}
& f^{(v)}(z) \equiv \frac{d^{v} f}{d z^{v}} \equiv D^{v} f(z) \equiv \frac{\Gamma(v+1)}{2 \pi i} \int_{\partial \mathbb{D}}(\xi-z)^{-v-1} f(\xi) d \xi \\
& \arg (z-\delta)<\arg (\xi)<\arg (z+\delta) ; f(\xi) \sim 0\left(\xi^{\Re(v)-\varepsilon}\right), \varepsilon>0 \text { for some } \delta>0 .
\end{aligned}
$$

Here in formula (3.1), $v$ is any complex number other than a negative integer, implying that the Gamma function $\Gamma(v+1)$ is analytic, as its poles; $v+1=0,-1,-2, \ldots$; have been excluded. The function $f(z)$ is a complex function of a complex variable $z$, analytic in the (open) interior $\mathbb{D}-\partial \mathbb{D}$ of the (closed) region of integration $\mathbb{D}$, which may or may not include the point-at-infinity; the boundary $\partial \mathbb{D}$ is a simple and regular (or rectifiable) curve, and on it the function $f(\xi)$ satisfies one of the progressively less stringent conditions that it is analytic and it is uniformly continuous $f(z) \rightarrow f(\xi)$ as $z$ in $\mathbb{D}-\partial \mathbb{D}$ tends to $\xi$ on $\mathbb{D}$; it is bounded on $\partial D$, with at most a finite number of discontinuities.

The Cauchy kernel $(\xi-z)^{-v-1}$ is single valued if $v$ is an integer, and the principal branch of this many-valued function is taken if $v$ is not an integer. The contour of integration $\partial \mathbb{D}$ is described in the positive (counterclockwise) direction around $z$, and does not cross any branch-cuts in the $\xi$-plane; if any of the branch-cuts is infinite, the contour $\partial \mathbb{D}$ must be open, and can only touch the cut at the point at infinity; if there are no branch-cuts, or all are finite, the contour $\partial \mathbb{D}$ is closed and finite (i.e. a loop), and may or may not touch a branchpoint, as necessary to ensure that the integrand returns to the initial value after describing the loop; the integral should be independent of the contour (provided it is deformed without crossing branch-cuts or touching any new branch-points and $\mathrm{z}$ remains in its interior), and uniformly convergent with regard to $\mathrm{z}$.

In a case, when we set $v=+n, \forall n \in \mathbb{Z}^{+}$, in (3.1), it becomes the Cauchy (1825) integral theorem, given by Campos (Campos, 1984, Eqn. (2), p. 114)

$$
f^{(n)}(z) \equiv \frac{d^{n} f}{d z^{n}} \equiv D^{n} f(z) \equiv \frac{n !}{2 \pi i} \int^{\left(z^{+}\right)}(\xi-z)^{-n-1} f(\xi) d \xi
$$

(See also, (Conway, 1973), (Oldham \& Spanier, 2006) and (Pathan \& Kumar, 2019)).

In another case, when a function $f(z)$ with a branch - point at $z=z_{0}$ and with complex exponent $\mu$, not an integer, that is $f(z)=\left(z-z_{0}\right)^{\mu} g(z), g(z)$ is analytic, then the derivative of complex order $v$ of complex function $f(z)$ of complex variable $z$, is defined by an Pochhammer's type integral as (see Campos (Campos, 1984, Eqn. (13), p. 118))

$$
\frac{d^{v}\left\{\left(z-z_{0}\right)^{\mu} g(z)\right\}}{d z^{v}} \equiv(4 \pi)^{-1} e^{-i \pi \mu} \frac{\Gamma(v+1)}{\sin \mu \pi} \int^{\left(z^{+}, z_{0}^{+}, z^{-}, z_{0}^{-}\right)}\left(\xi-z_{0}\right)^{\mu} g(\xi)(\xi-z)^{-v-1} d \xi,
$$

$\arg (z-\delta)<\arg (\xi)<\arg (z+\delta) ; \lim _{\xi \rightarrow \infty} \xi^{m} f(\xi)=0$, some $\delta>0$ and for all $m$.

Theorem 3.1. If $G(\xi, z)=\lim _{\xi \rightarrow z}\left\{\frac{\Omega_{m}^{(\alpha)}(\xi)-\Omega_{m}^{(\alpha)}(z)}{\xi-z}\right\}=A^{(\alpha)}=\left\{\log _{e} E_{\alpha}\right\}^{-1}$, and the equation $\Omega_{m}^{(\alpha)}(\xi)-\Omega_{m}^{(\alpha)}(z)=0$ has single root $\xi=z$ in $\mathbb{D}, \Omega_{m}^{(\alpha)}(z)$ is given in (2.10), and the function $f: \mathbb{D} \rightarrow \mathbb{C}$, defined by $f(z)=\left(z-z_{0}\right)^{\mu} g(z) \forall z, z_{0} \in \mathbb{C}, g(z)$ is analytic in complex $\xi$-plane, then $\forall v, \mu \in \mathbb{C}$, there exists a extended Pochhammer's type contour integral formula for complex $v$ order derivative with respect to the function $\Omega_{m}^{(\alpha)}(z)$ as 


$$
\begin{aligned}
& \frac{d^{v}\left\{\left(z-z_{0}\right)^{\mu} g(z)\right\}}{d\left\{\Omega_{m}^{(\alpha)}(z)\right\}^{v}} \\
& \quad=\frac{e^{-i \pi \mu}}{4 \pi} \frac{\Gamma(v+1)}{\sin \mu \pi}\left\{\log _{e} E_{\alpha}\right\}^{v} \int^{\left(z^{+}, z_{0}^{+}, z^{-}, z_{0}^{-}\right)}\left(\xi-z_{0}\right)^{\mu} g(\xi)(\xi-z)^{-v-1} d \xi .
\end{aligned}
$$

Proof. By the rulings of (3.1) and (3.3), we define

$$
\begin{aligned}
& \frac{d^{v}\left\{\left(z-z_{0}\right)^{\mu} g(z)\right\}}{d\left\{\Omega_{m}^{(\alpha)}(z)\right\}^{v}}=\frac{e^{-i \pi \mu}}{4 \pi} \frac{\Gamma(v+1)}{\sin \mu \pi} \\
& \times \lim _{\xi \rightarrow z} \int^{\left(z^{+}, z_{0}^{+}, z^{-}, z_{0}^{-}\right)}\left(\xi-z_{0}\right)^{\mu} g(\xi)(\xi-z)^{-v-1}\{G(\xi, z)\}^{-v-1}\left\{\Omega_{m}^{(\alpha)}(\xi)\right\}^{\prime} d \xi \\
& =\frac{e^{-i \pi \mu}}{4 \pi} \frac{\Gamma(v+1)}{\sin \mu \pi} \\
& \times \lim _{\xi \rightarrow z} \int^{\left(z^{+}, z_{0}^{+}, z^{-}, z_{0}^{-}\right)}\left(\xi-z_{0}\right)^{\mu} g(\xi)(\xi-z)^{-v-1}\left\{\frac{\Omega_{m}^{(\alpha)}(\xi)-\Omega_{m}^{(\alpha)}(z)}{\xi-z}\right\}^{-v-1}\left\{\Omega_{m}^{(\alpha)}(\xi)\right\}^{\prime} d \xi .
\end{aligned}
$$

Then, in it apply the results due to (2.10), the Theorem 2.1 and (1.1).Thus, we get the result

$$
\begin{aligned}
& \frac{d^{v}\left\{\left(z-z_{0}\right)^{\mu} g(z)\right\}}{d\left\{\Omega_{m}^{(\alpha)}(z)\right\}^{v}} \\
& \quad=\frac{e^{-i \pi \mu}}{4 \pi} \frac{\Gamma(v+1)}{\sin \mu \pi} \lim _{\xi \rightarrow z} \int^{\left(z^{+}, z_{0}^{+}, z^{-}, z_{0}^{-}\right)}\left(\xi-z_{0}\right)^{\mu} g(\xi)(\xi-z)^{-v-1}\left\{A^{(\alpha)}\right\}^{-v} d \xi .
\end{aligned}
$$

By making use of this result, we easily obtain (3.4). This completes the proof of the Theorem.

Remark 3.1. To verify above result (3.4), put $\alpha=1$ in formula (3.4) and use the property of $E_{\alpha}$ in (1.3) for $\log _{e} E_{\alpha}$ as $\left.\log _{e} E_{\alpha}\right|_{\alpha=1}=1$, then $A^{(\alpha)}=1$ and $\Omega_{m}^{(\alpha)}(z)=z$ and hence, this formula becomes equivalent to the formula (3.3), (see Campos (Campos, 1984)).

\section{An application to obtain the extended Pochhammer's type inte- gral representations and Rodrigues formulae of hypergeomet- ric functions}

In this section, we apply our result (3.4) of the Theorem 3.1 and obtain the extended Pochhammer's type integral representations and Rodrigues formulae of various known hypergeometric functions.

To achieve our goal, we present an integral representation of Kummer's function ${ }_{1} F_{1}$ [.] by Erdelyi (ErdImagelyi, Magnus, Oberhettinger, \& Tricomi, 1953, Vol. 1, p. 271) due to Campos (Campos, 1984, Eqn. (37) with (36b)) as the Pochhammer type contour integral given by

$$
{ }_{1} F_{1}[b ; c ; z]=B \int^{\left(z^{+}, 0^{+}, z^{-}, 0^{-}\right)} \xi^{b-1}(\xi-z)^{c-b-1} e^{\xi} d \xi,
$$

where, $B=\frac{1}{4 \pi^{2}} e^{-i b \pi} \Gamma(1-b) \Gamma(c) \Gamma(1+b-c) z^{1-c}$.

Now in (3.4), put $\mu=b-1, v=b-c, z_{0}=0, g(z)=e^{z}$, the complex $b-1$ is not an integer, and thus multiply both of the sides by the function $\Gamma(1-b) \Gamma(c) z^{1-c} \frac{\sin b \pi}{\pi}$, and use the results (2.10) and (1.1), and compare with (4.1) to get 


$$
\begin{array}{r}
\Gamma(1-b) \Gamma(c) z^{1-c} \frac{\sin b \pi}{\pi} \frac{d^{b-c}\left(z^{b-1} e^{z}\right)}{d\left\{\Omega_{m}^{(\alpha)}(z)\right\}^{b-c}}=\frac{\Gamma(c)}{\Gamma(b)} z^{1-c} \frac{d^{b-c}\left(z^{b-1} e^{z}\right)}{d\left\{\Omega_{m}^{(\alpha)}(z)\right\}^{b-c}}={ }_{1}^{(\alpha)} F_{1}[b ; c ; z] \text { (let) } \\
=e^{-i \pi b} \Gamma(1-b) \Gamma(c) \frac{\Gamma(b-c+1)}{4 \pi^{2}} z^{1-c}\left\{\log _{e} E_{\alpha}\right\}^{b-c} \int^{\left(z^{+}, z_{0}^{+}, z^{-}, z_{0}^{-}\right)}(\xi)^{b-1} e^{\xi}(\xi-z)^{c-b-1} d \xi \\
=\left\{\log _{e} E_{\alpha}\right\}_{1}^{b-c} F_{1}[b ; c ; z] . \quad \text { (4.2) }
\end{array}
$$

Therefore, the extended Pochhammer type contour integral representation of Kummer's type hypergeometric function ${ }_{1}^{(\alpha)} F_{1}[b ; c ; z]$ is of the form

$$
\begin{gathered}
\quad{ }_{1}^{(\alpha)} F_{1}[b ; c ; z]=\frac{\Gamma(c)}{\Gamma(b)} z^{1-c} \frac{d^{b-c}\left(z^{b-1} e^{z}\right)}{d\left\{\Omega_{m}^{(\alpha)}(z)\right\}^{b-c}} \\
=e^{-i \pi b} \Gamma(1-b) \Gamma(c) \frac{\Gamma(b-c+1)}{4 \pi^{2}} z^{1-c}\left\{\log _{e} E_{\alpha}\right\}^{b-c} \int^{\left(z^{+}, z_{0}^{+}, z^{-}, z_{0}^{-}\right)}(\xi)^{b-1} e^{\xi}(\xi-z)^{c-b-1} d \xi .
\end{gathered}
$$

Remark 4.1. Setting $\alpha=1$ in the result (4.3), we find the results of Compose (Campos, 1984, Eqn. (37) with (36b)).

Again in (3.4), set $\mu=b-1, v=b-c, z_{0}=0, g(z)=(1-\sigma z)^{-\gamma}(1-\rho z)^{-\beta}$, the complex $b-1$ is not an integer, $|\sigma z|<1,|\rho z|<1$ and then multiply both the sides by the function $\Gamma(1-b) \Gamma(c) z^{1-c} \frac{\sin b \pi}{\pi}$. Now use the results of (4.2) together with the formula (Srivastava and Manocha (H. M. Srivastava \& Manocha, 1984, p.290)) to obtain the Pochhammer contour integral of Appell's function $F_{1}[.,$.$] of two variables, given by$

$$
\begin{gathered}
{ }^{(\alpha)} F_{1}[b, \gamma, \beta ; c ; \sigma z, \rho z]=\frac{\Gamma(c)}{\Gamma(b)} z^{1-c} \frac{d^{b-c}\left(z^{b-1}(1-\sigma z)^{-\gamma}(1-\rho z)^{-\beta}\right)}{d\left\{\Omega_{m}^{(\alpha)}(z)\right\}^{b-c}} \\
=e^{-i \pi b} \Gamma(1-b) \Gamma(c) \frac{\Gamma(b-c+1)}{4 \pi^{2}} z^{1-c}\left\{\log _{e} E_{\alpha}\right\}^{b-c} \\
\times \int^{\left(z^{+}, z_{0}^{+}, z^{-}, z_{0}^{-}\right)}(\xi)^{b-1}(1-\sigma \xi)^{-\gamma}(1-\rho \xi)^{-\beta}(\xi-z)^{c-b-1} d \xi \\
=\left\{\log _{e} E_{\alpha}\right\}^{b-c} F_{1}[b, \gamma, \beta ; c ; \sigma z, \rho z] .
\end{gathered}
$$

Further, put $\sigma=1, \rho=0$ in Eqn. (4.4), to find the Pochhammer's type contour integral formula for the Gaussian hypergeometric function ${ }_{2} F_{1}[$.$] , given by$

$$
\begin{aligned}
&{ }_{2}^{(\alpha)} F_{1}[b, \alpha ; c ; z]= \frac{\Gamma(c)}{\Gamma(b)} z^{1-c} \frac{d^{b-c}\left(z^{b-1}(1-z)^{-\gamma}\right)}{d\left\{\Omega_{m}^{(\alpha)}(z)\right\}^{b-c}} \\
&=e^{-i \pi b} \Gamma(1-b) \Gamma(c) \frac{\Gamma(b-c+1)}{4 \pi^{2}} z^{1-c}\left\{\log _{e} E_{\alpha}\right\}^{b-c} \\
& \times \int^{\left(z^{+}, z_{0}^{+}, z^{-}, z_{0}^{-}\right)}(\xi)^{b-1}(1-\sigma \xi)^{-\alpha}(1-\xi)^{-\gamma}(\xi-z)^{c-b-1} d \xi \\
&=\left\{\log _{e} E_{\alpha}\right\}^{b-c}{ }_{2} F_{1}[b, \gamma ; c ; z] .
\end{aligned}
$$

Remark 4.2. Setting $\alpha=1$ in the result (4.5), we find the results of Compose (Campos, 1984, Eqn. (32) with (31b)). 


\section{An application in the case of spread of infectious disease in terms of multiple hypergeometric functions}

In this section, we consider another property of the logarithmic Mittag - Leffler function to obtain the area of spread of infectious disease for different parameters and weight functions. The results are evaluated in terms of Lauricella's multiple hypergeometric functions.

Theorem 5.1. If $G(z)=e^{-z} \log E_{\frac{1}{m}}\left(z^{\frac{1}{m}}\right)$, (given in Lemma 2.3) and $e^{-z} z^{-\frac{1}{m}} F(z) \in L_{1}(\mathbb{R})$, $\forall z \in \mathbb{C},, m \in \mathbb{N}^{*}, \mathbb{N}^{*}=\{2,3,4, \ldots, L\}, L<\infty$, there exists a function $F: \mathbb{R} \rightarrow \mathbb{R}$, defined by $\Delta=\int_{R} F(\xi)\left(\frac{d}{d \xi}\left\{\exp \left[e^{\xi} G(\xi)-\xi\right]\right\}\right) d \xi$, and then

$$
\Delta=\sum_{r=1}^{m-1} \frac{1}{\Gamma\left(1-\frac{r}{m}\right)} \int_{R} e^{-\xi} \xi^{-\frac{r}{m}} F(\xi) d \xi
$$

Proof. Since $G(z)=e^{-z} \log E_{\frac{1}{m}}\left(z^{\frac{1}{m}}\right)$,we may write

$$
\log E_{\frac{1}{m}}\left(z^{\frac{1}{m}}\right)=e^{z} G(z) \Rightarrow e^{-z} E_{\frac{1}{m}}\left(z^{\frac{1}{m}}\right)=\exp \left[e^{z} G(z)-z\right]
$$

so that on using the formula (see Mathai and Haubold (Mathai \& Haubold, 2008, p. 84)),

$$
\frac{d}{d z}\left(e^{-z} E_{\frac{1}{m}}\left(z^{\frac{1}{m}}\right)\right)=e^{-z} \sum_{r=1}^{m-1} \frac{z^{-\frac{r}{m}}}{\Gamma\left(1-\frac{r}{m}\right)}
$$

we may further write as

$$
\frac{d}{d z} \exp \left[e^{z} G(z)-z\right]=e^{-z} \sum_{r=1}^{m-1} \frac{z^{-\frac{r}{m}}}{\Gamma\left(1-\frac{r}{m}\right)}
$$

Thus we have

$$
\int_{R} F(\xi)\left(\frac{d}{d \xi}\left\{\exp \left[e^{\xi} G(\xi)-\xi\right]\right\}\right) d \xi=\sum_{r=1}^{m-1} \frac{1}{\Gamma\left(1-\frac{r}{m}\right)} \int_{R} e^{-\xi} \xi-\frac{r}{m} F(\xi) d \xi
$$

which gives the result (5.1).

Theorem 5.2. Let the infectious disease is spreading by the rulings $F(x)=\left(\frac{d}{d x}\left\{\exp \left[e^{x} G(x)-\right.\right.\right.$ $x]\}), x>0$, where, $G(x)=e^{-x} \log E_{\frac{1}{m}}\left(x^{\frac{1}{m}}\right) ; \forall m \in \mathbb{N}^{*}, \mathbb{N}^{*}=\{2,3,4, \ldots, L\}, L<\infty$; with the weight function $P(x)=e^{x} \prod_{j=1}^{n}\left(\left(\rho_{0}-a_{j} x\right)\right)^{-b_{j}}$, and the parameters $\rho_{0}>0, b_{j} \in \mathbb{R}, 0<$ $a_{j}<1 \forall j=1,2,3, \ldots, n$; then, for $\rho>\rho_{0}$, the spreading area $\Delta$ of the disease in the domain $\left(\rho_{0}, \rho\right) \in \mathbb{R}$, is obtained by the formula $\Delta=\int_{\rho_{0}}^{\rho} P(x)\left(\frac{d}{d x}\left\{\exp \left[e^{x} G(x)-x\right]\right\}\right) d x$ and found by

$$
\begin{aligned}
& \Delta=\sum_{r=1}^{m-1} \frac{\left(\rho-\rho_{0}\right) \prod_{j=1}^{n}\left(\rho_{0}\left(1-a_{j}\right)\right)^{-b_{j}}}{\Gamma\left(1-\frac{r}{m}\right)}\left(\rho_{0}\right)^{-\frac{r}{m}} \sum_{k=0}^{\infty} \frac{\left(\frac{r}{m}\right)_{k}}{(k+1) !}\left(-\left\{\frac{\left(\rho-\rho_{0}\right)}{\rho_{0}}\right\}\right)^{k} \\
& \times F_{D}^{(n)}\left(k+1, b_{1}, \ldots, b_{n} ; k+2 ; \frac{\left(\left(\rho-\rho_{0}\right) a_{1}\right)}{\left(\rho_{0}\left(1-a_{1}\right)\right)}, \ldots, \frac{\left(\left(\rho-\rho_{0}\right) a_{n}\right)}{\left(\rho_{0}\left(1-a_{n}\right)\right)}\right) .
\end{aligned}
$$

Here, the $F_{D}^{(n)}($.$) being a Lauricella's multiple hypaergeometric function defined in (\boldsymbol{H}$. M. Srivastava \& Manocha, 1984, Eqn.(4),p. 60).

Proof. Starting from (5.1), we have 


$$
\begin{array}{r}
\Delta=\sum_{r=1}^{m-1} \frac{1}{\Gamma\left(1-\frac{r}{m}\right)} \int_{\rho_{0}}^{\rho} e^{-x} x^{-\frac{r}{m}} P(x) d x=\sum_{r=1}^{m-1} \frac{1}{\Gamma\left(1-\frac{r}{m}\right)} \int_{\rho_{0}}^{\rho} x^{-\frac{r}{m}} \prod_{j=1}^{n}\left(\rho_{0}-a_{j} x\right)^{-b_{j}} d x \\
\forall m \in \mathbb{N}^{*}, \mathbb{N}^{*}=\{2,3,4, \ldots, L\}, L<\infty .
\end{array}
$$

Now suppose $0<\rho_{0}<\rho, x-\rho_{0}=\left(\rho-\rho_{0}\right) t \forall 0<\rho_{0} \leq x \leq \rho, t \geq 0^{+}$, then

$$
\begin{gathered}
\Delta=\sum_{r=1}^{m-1} \frac{\left(\rho-\rho_{0}\right) \prod_{j=1}^{n}\left(\rho_{0}\left(1-a_{j}\right)\right)^{-b_{j}}}{\Gamma\left(1-\frac{r}{m}\right)} \int_{0}^{1}\left(\rho_{0}+\left(\rho-\rho_{0}\right) t\right)^{-\frac{r}{m}} \prod_{j=1}^{n}\left(1-\frac{\left(\left(\rho-\rho_{0}\right) a_{j}\right)}{\left(\rho_{0}\left(1-a_{j}\right)\right)} t\right)^{-b_{j}} d t \\
\Rightarrow \Delta=\sum_{r=1}^{m-1} \frac{\left(\rho-\rho_{0}\right) \prod_{j=1}^{n}\left(\rho_{0}\left(1-a_{j}\right)\right)^{-b_{j}}}{\Gamma\left(1-\frac{r}{m}\right)}\left(\rho_{0}\right)^{-\frac{r}{m}} \sum_{k=0}^{\infty} \frac{\left(\frac{r}{m}\right)_{k}}{k !}\left(-\left\{\frac{\left(\rho-\rho_{0}\right)}{\rho_{0}}\right\}\right)^{k} \\
\times \int_{0}^{1} t^{k+1-1}(1-t)^{k+2-(k+1)-1} \prod_{j=1}^{n}\left(1-\frac{\left(\left(\rho-\rho_{0}\right) a_{j}\right)}{\left(\rho_{0}\left(1-a_{j}\right)\right)} t\right)^{-b_{j}} d t
\end{gathered}
$$

which on applying the technique of Chandel, Agrawal and Kumar (Chandel, Agrawal, \& Kumar, 1993), and the formula of the Exton (Exton, 1976, Eqn. (2.3.6), p. 49) yields the result (5.2).

Remark 5.1. In the Theorem 5.2, we have used the ruling,

$$
F(x)=\left(\frac{d}{d x}\left\{\exp \left[e^{x} G(x)-x\right]\right\}\right) \text { and } G(x)=e^{-x} \log E_{\frac{1}{m}}\left(x^{\frac{1}{m}}\right),
$$

which emphasize the following useful integral

$$
\int_{t_{0}}^{t} F(x) d x=E_{\frac{1}{m}}\left(t^{\frac{1}{m}}\right) e^{-t}-E_{\frac{1}{m}}\left(t_{0}^{\frac{1}{m}}\right) e^{-t_{0}} \forall m \in \mathbb{N}^{*}, \mathbb{N}^{*}=\{2,3,4, \ldots, L\}, L<\infty .
$$

Theorem 5.3. Let the infectious disease is spreading by the rulings $F(x)=\left(\frac{d}{d x}\left\{\exp \left[e^{x} G(x)-\right.\right.\right.$ $x]\}), x>0$, where, $G(x)=e^{-x} \log E_{\frac{1}{m}}\left(x^{\frac{1}{m}}\right) ; \forall m \in \mathbb{N}^{*}, \mathbb{N}^{*}=\{2,3,4, \ldots, L\}, L<\infty$; with the weight function $P(x)=\prod_{j=1}^{n}{ }_{1} F_{1}\left(b_{j} ; c_{j} ; a_{j} x\right),|x|<\infty$ and the parameters $b_{j} \in \mathbb{R}, a_{j}>0, c_{j} \neq$ $0,-1,-2, \ldots, \forall j=1,2,3, \ldots, n$, and $\left|a_{1}+\ldots+a_{n}\right|<1$. Then the spreading area $\Delta$ of the disease in the domain $(0, \infty) \subset \mathbb{R}$, is obtained by the formula $\Delta^{\prime}=\int_{0}^{\infty} P(x)\left(\frac{d}{d x}\left\{\exp \left[e^{x} G(x)-\right.\right.\right.$ $x]\}) d x$ and found by

$$
\Delta^{\prime}=\sum_{r=1}^{m-1} F_{A}^{(n)}\left(1-\frac{r}{m}, b_{1}, \ldots, b_{n} ; c_{1}, \ldots, c_{n} ; a_{1}, \ldots, a_{n}\right) .
$$

Proof. In the similar manner of Theorem 5.2, we have

$$
\begin{aligned}
\Delta^{\prime}=\int_{0}^{\infty} P(x)\left(\frac{d}{d x}\left\{\exp \left[e^{x} G(x)-x\right]\right\}\right) & d x \\
& =\sum_{r=1}^{m-1} \frac{1}{\Gamma\left(1-\frac{r}{m}\right)} \int_{0}^{\infty} e^{-x} x^{-\frac{r}{m}} \prod_{j=1}^{n}{ }_{1} F_{1}\left(b_{j} ; c_{j} ; a_{j} x\right) d x .
\end{aligned}
$$

Then apply the Euler integral formula of Exton (Exton, 1976, Eqn. (2.4.2), p. 49)

$$
\begin{aligned}
F_{A}^{(n)}\left(a, b_{1}, \ldots, b_{n} ; c_{1}, \ldots, c_{n} ; x_{1}, \ldots,\right. & \left.x_{n}\right) \\
& =\frac{1}{\Gamma(a)} \int_{0}^{\infty} e^{-t} t^{a-1} \prod_{j=1}^{n}{ }_{1} F_{1}\left(b_{j} ; c_{j} ; x_{j} t\right) d t, \Re(a)>0,
\end{aligned}
$$

to get the formula (5.4).The $F_{A}^{(n)}($.$) being a Lauricella's multiple hypaergeometric function$ defined in (H. M. Srivastava \& Manocha, 1984, Eqn. (1), p. 60). 


\section{Relations of logarithmic Mittag Leffler function into Riemann- Liouville fractional integral}

From the Theorem 5.1, consider that

$G(z)=e^{-z} \log E_{1}\left(z^{\frac{1}{m}}\right), m=2,3,4, \ldots$, and that the Riemann - Liouville fractional integral, given by (Diethelm, 2010, p.13)

$$
I_{0}^{v} \varphi(z)=\frac{1}{\Gamma(v)} \int_{0}^{z}(z-\zeta)^{v-1} \varphi(\zeta) d \zeta, v>0
$$

Therefore by proof of the Theorem 5.1, we have

$$
e^{z} \frac{d}{d z} \exp \left[e^{z} G(z)-z\right]=\sum_{r=1}^{m-1} \frac{z^{-\frac{r}{m}}}{\Gamma\left(1-\frac{r}{m}\right)} .
$$

Now, define that

$$
H^{(v)}(z)=\left\{\begin{array}{c}
\frac{z^{v}}{\Gamma(v)}, 0<z<\infty, v>0 \\
0, \text { elsewhere }
\end{array}\right.
$$

and any function $\varphi(z)$ such that $\lim _{z \rightarrow \pm \infty} \varphi(z)=0$.

Then for $z>0$, make an appeal to the Theorem 5.1 and along with formulae (5.4) and (5.5), we obtain the relation of logarithmic function with the Riemann - Liouville fractional integral as

$$
\begin{aligned}
\int_{-\infty}^{\infty} e^{\zeta} \varphi(z-\zeta) & \frac{d}{d \zeta} \exp \left[e^{\zeta} G(\zeta)-\zeta\right] d \zeta=\sum_{r=1}^{m-1} \int_{0}^{z} H^{\left(1-\frac{r}{m}\right)}(z-\zeta) \varphi(\zeta) d \zeta \\
= & \sum_{r=1}^{m-1} \int_{0}^{z}(z-\zeta)^{\left(1-\frac{r}{m}\right)-1} \varphi(\zeta) d \zeta=\sum_{r=1}^{m-1} I_{0}^{\left(1-\frac{r}{m}\right)} \varphi(z) \forall m=2,3,4, \ldots
\end{aligned}
$$

In another way, we write

$$
\begin{array}{r}
\left(e^{\zeta} \varphi(z-\zeta) \exp \left[e^{\zeta} G(\zeta)-\zeta\right]\right) \underset{\zeta=-\infty}{\zeta=\infty}-\int_{-\infty}^{\infty} \exp \left[e^{\zeta} G(\zeta)-\zeta\right] \frac{d}{d \zeta}\left\{e^{\zeta} \varphi(z-\zeta)\right\} d \zeta \\
=\sum_{r=1}^{m-1} \int_{0}^{z}(z-\zeta)^{\left(1-\frac{r}{m}\right)-1} \varphi(\zeta) d \zeta=\sum_{r=1}^{m-1} I_{0}^{\left(1-\frac{r}{m}\right)} \varphi(z) \forall m=2,3,4, \ldots
\end{array}
$$

Therefore, we get

$$
\begin{aligned}
\int_{-\infty}^{\infty} \exp \left[e^{\zeta} G(\zeta)\right]\left\{\left(1+\frac{d}{d \zeta}\right) \varphi(z-\zeta)\right\} d \zeta & =-\sum_{r=1}^{m-1} \int_{0}^{z} H^{\left(1-\frac{r}{m}\right)}(z-\zeta) \varphi(\zeta) d \zeta \\
& =-\sum_{r=1}^{m-1} I_{0}^{\left(1-\frac{r}{m}\right)} \varphi(z) \forall m=2,3,4, \ldots
\end{aligned}
$$

\section{Conclusions}

The ideas and techniques employed for the Mittag-Leffler function can be used for numerical calculation of other functions of the hypergeometric type (see for example ((Gorenflo et al., 2002) and (Peng $\& \mathbf{L i}, 2010)$ ). In particular, the same method with some small modifications can be applied for the Wright function playing a very important role in the 
theory of partial differential equations of fractional order (see for example, (Gorenflo et al., 1999)).To this end, simple examples of Mittag - Leffler function are well known. Some of the more complicated examples on logarithmic Mittag - Leffler function do not seem to be well known. In our investigation, we defined the logarithmic Mittag - Leffler function with prescribed conditions and discussed some of its analytic properties. In order to illustrate the application of the general properties, we have demonstrated the usefulness of the concept, in connection with the theory of special functions, their extended Pochhammer's type integral representations, Rodrigues formulae and fractional calculus. In certain conditions, it becomes very useful in obtaining complex order derivative to develop the uses of special functions, their Rodrigues formulae and fractional calculus. The results obtained in our paper are involving Kummer hypergeometric function, Gaussian hypergeometric function and Appell's function. By manipulation of their parameters, we may find Bessel's function, Laguerre function, Legendre function, Hermite function, Tchebycheff function and Gegenbauer function of complex order on the lines of the work found in the literature of Campos (Campos, 1984).

\section{Acknowledgments}

We are thankful to the Editor and reviewer for their valuable suggestions to bring the paper it its present form.

\section{Author contributions}

In this paper, both authors have equally contributed in the recent researches.

\section{Conflicts of interest}

The authors declare no potential conflict of interests.

\section{References}

Campos, L. M. B. C. (1984). On a concept of derivative of complex order with applications to special functions. IMA J. Appl. Math., 33(), 109-133.

Caponetto, R., Dongola, G., \& L. Fortuna, I. P. (2010). Fractional order systems: Modeling and control applications. World Scientific, Singapore.

Chandel, R. C. S., Agrawal, R. D., \& Kumar, H. (1993). Velocity coefficient of chemical reaction and lauricella's function $f_{D}^{(n)}$, . Math. Student, 62(1-4), 93-96.

Conway, J. B. (1973). Functions of one complex variable. Springer International Student Edition, Springer-Verlag.

Diethelm, K. (2010). The analysis of differential equations of fractional order: An application-oriented exposition using differential operators of caputo type, lecture notes in mathematics (Vol. 2004). Springer Verlag: Berlin-Heidelberg, Germany.

Dzherbashian, M. M. (1966). Integral transforms and representation of functions in the complex domain. Nauka: Moscow, Russian, (In Russian).

ErdImagelyi, A., Magnus, W., Oberhettinger, F., \& Tricomi, F. G. (1953). Higher transcendental functions (Vol. I). McGraw-Hill, New York.

Exton, H. (1976). Multiple hypergeometric functions and applications. Ellis Horwood Limited, England.

Gorenflo, R., Kilbas, A. A., Mainardi, F., \& Rogosin, S. (2014). Mittag-leffler functions, related topics and applications. Springer-Verlag:Berlin-Heidelberg, Geramny.

Gorenflo, R., Loutchko, J., \& Luchko, Y. (2002). Computation of the mittag - leffler function image. Fract. Calc. Appl. Anal., 5(4), 491-518.

Gorenflo, R., Luchko, Y., \& Mainardi, F. (1999). Analytical properties and applications of the wright function. Fract. Calc. Appl. Anal., 2(), 383-414. 
Gorenflo, R., Mainardi, F., \& Srivastava, H. M. (1998). Special functions in fractional relaxation oscillation and fractional diffusion-wave phenomena. VSP Publishers: Utrecht, The Netherlands; Tokyo, Japan.

Hilfer, R. E. (2000). Applications of fractional calculus in physics. World Scientific: Singapore.

Kilbas, A. A., Srivastava, H. M., \& Trujillo, J. J. (2006). Theory and applications of fractional differential equations (Vol. 204). Elsevier: Amsterdam, The Netherlands.

Kiryakova, V. (1999). Multi-index mittag-leffler functions, related gelfond- leontiev operators and laplace type integral transforms. Fract. Calc. Appl. Anal., 2(), 445-462.

Kiryakova, V., \& Luchko, Y. (2010). The multi-index mittag-leffler functions and their applications for solving fractional order problems in applied analysis. in application of mathematics in technical and natural sciences (cp1301, amitans'10); todorov, m.d., christov, c.i., eds. American Institute of Physics: Melville, NY, USA.

Mathai, A. M., \& Haubold, H. J. (2008). Special functions for applied scientists. Springer, New York.

Mittag-Leffler, G. M. (1903). Sur la nonvelle function $e_{\alpha}(x)$. C. R. Acad. Paris, 137(), 554-558.

Oldham, K. B., \& Spanier, J. (2006). The fractional calculus, theory and applications of differentiation and integration to arbitrary order. Dover Publication, Inc., New York.

Osler, T. J. (1972). A further extension of the leibniz rule to fractional derivatives and its relation to parseval's formula. SIAM J., Math. Anal., 3(1), 1-16.

Pathan, M. A., \& Kumar, H. (2019). Generalized multivariable cauchy residue theorem and nonzero zeros of multivariable and multi-parameters generalized mittag-leffler functions. Southeast Asian Bull. Math., 43(), 733-749.

Peng, J., \& Li, K. (2010). A note on property of the mittag-leffler function. J. Math. Anal. Appl., 370(), 635-638.

Podlubny, I. (1999). Fractional differential equations. Academic Press: New York, NY, USA.

Rogosin, S. (2015). The role of the mittag - leffler function in fractional modeling. Mathematics, 3(), 368-381.

Samko, S. G., Kilbas, A. A., \& Marichev, O. I. (1993). Fractional integrals and derivatives: Theory and applications.

Shukla, A. K., \& Prajapati, J. C. (2007). On a generalization of mittag-leffler function and its properties. J. Math. Anal. Appl., 336(), 797-811.

Srivastava, B. L., Pathan, M. A., \& Kumar, H. (2020). An abelian group of a class of mittag - leffler functions. Romanian J. Math. Comput. Sci., 10(1)(), 67-72.

Srivastava, H. M., \& Manocha, H. L. (1984). A treatise on generating functions. John Wiley and Sons, New York.

Steele, J. M. (2004). The cauchy - schwarz master class; a introduction to the art of mathematical inequalities. Cambridge University Press Cambridge, New York.

Wiman, A. (1905). Uber den fundamental saiz in der theorie der funktionen image. Acta. Math., 29(), 191-201. 